\title{
Diseño e Implementación de Kits para la Experimentación de Biotecnología en el Hogar (HomeLab)
}

\author{
A. L 'Torres-Huerta ${ }^{a}$, R.V. Gómez-Acata ${ }^{b}$, M. Jaime-Rodríguez ${ }^{\mathfrak{c}}$, Y. G. García-Huante ${ }^{\mathrm{d}}$, J. I. \\ Calzada-Frías ${ }^{\mathrm{e}}$ E. O. Gómez-Montes ${ }^{\mathrm{f}}$, R. Delgado-Durán ${ }^{\mathrm{g}}$, C. García-Díaz ${ }^{\mathrm{h}}$, C. E. Gómez-Sánchezi, \\ A. Antonio Pérez. \\ a Profesor planta, Tecnológico de Monterrey, atorresh@tec.mx (autor de correspondencia), b Profesor planta, \\ Tecnológico de Monterrey, Escuela de Ingeniería y Ciencias, rigel@tec.mx , c Profesor cátedra, Tecnológico de \\ Monterrey, Escuela de Ingeniería y Ciencias, mjaimer@tec.mx, ${ }^{\mathrm{d}}$ Profesor cátedra, Tecnológico de Monterrey, \\ Escuela de Ingeniería y Ciencias, yori gh12@tec.mx, ${ }^{\text {e }}$ Profesor cátedra, Tecnológico de Monterrey, Escuela de \\ Ingeniería y Ciencias, jcalzada@,tec.mx , ${ }^{\mathrm{f}}$ Profesor cátedra, Tecnológico de Monterrey, Escuela de Ingeniería y \\ Ciencias, octavio.gomez@tec.mx, ${ }^{g}$ Profesor y Responsables de Laboratorios, Tecnológico de Monterrey, Escuela de \\ Ingeniería y Ciencias, robertoduran@tec.mx, ${ }^{h}$ jefe de Carrera IBT, Tecnológico de Monterrey, cgarciad@tec.mx, ${ }^{i}$ \\ jefe del Departamento de Bioingeniería, Tecnológico de Monterrey, Escuela de Ingeniería y Ciencias, \\ carlos.eduardo.gomez@tec.mx,${ }^{j}$ Profesor planta, Tecnológico de Monterrey, Escuela de Ingeniería y Ciencias, \\ a.antonio@tec.mx (autor de correspondencia).
}

\begin{abstract}
With the situation of the COVID-19 pandemic, professors had to accelerate their adaptation to the use of digital and remote media to continue with the education of students. Online teaching lab courses present great challenges, as many lab courses are designed to learn from hands-on and real-world exploration. Laboratory courses focus on deepening understanding of content and developing skills in experimental techniques. Added to this, the possibility of providing the experience of executing protocols and manipulating equipment, can hardly be achieved with a completely digital model. To promote better learning of laboratory subjects, kits were developed by professors to conduct laboratory practices at home. The students had the opportunity to execute biotechnology protocols following instructions and remote guidance from their professors. Conducting experiments at home, ${ }^{l}$ had a high acceptance rate as an efficient tool to learn various skills compared to digital tools.
\end{abstract}

Keywords: home lab, kits, biotechnology, genetic engineering, enzymology, tissue culture, bioprocess.

\section{Resumen}

Con la situación de la pandemia COVID-19, la educación tuvo que acelerar su adaptación al uso de medios digitales y remotos para continuar con la educación de los estudiantes. Los cursos de laboratorio de enseñanza en línea presentan grandes desafios, ya que están diseñados para aprender de la exploración práctica y del mundo real. Sumado a esto, la posibilidad de brindar la experiencia de ejecutar protocolos y manipular equipos, dificilmente se puede lograr con un modelo completamente digital. Para promover un mejor aprendizaje y asegurar la adquisición de competencias de desarrollo de habilidades en técnicas experimentales a distancia, los profesores desarrollaron kits para la realización de prácticas de laboratorio en casa. Los estudiantes tuvieron la oportunidad de ejecutar protocolos de biotecnología siguiendo instrucciones y guía a distancia por parte de sus profesores. La realización de experimentos en casa tuvo un alto porcentaje de aceptación como una herramienta eficiente para aprender diversas competencias en comparación con herramientas digitales.

Palabras clave: laboratorio en casa, kits, Biotecnología, ingeniería genética, enzimología, cultivo de tejidos, bioprocesos 


\section{Introducción}

En respuesta al creciente número de casos de COVID-19, a mediados de marzo de 2020, las instituciones educativas tomaron medidas para cesar la enseñanza presencial, lo cual requirió de grandes esfuerzos para migrar a un modelo flexible-digital (Burki, 2020). Este cambio ha propiciado el diseño y uso de numerosas herramientas de enseñanza en línea y el desarrollo de aulas virtuales (Ray et al., 2020). Los programas de laboratorios de enseñanza de biotecnología se ven afectados por este cambio debido a la inaccesibilidad física a los mismos. La enseñanza de asignaturas de laboratorio mediante herramientas digitales es un gran reto, porque requiere la implementación de diversas estrategias, que difícilmente se pueden concretar de forma completa e integral en ambientes digitales, ya que se busca lograr el desarrollo de habilidades prácticas en los estudiantes (Gliddon et al., 2012).

Algunas universidades han puesto en marcha proyectos que intentan brindar a los estudiantes una experiencia de laboratorio (Monash University, 2020). La universidad La Trobe implementó laboratorios remotos de libre acceso, que consisten en una selección de experimentos científicos reales (manipular fuentes radiactivas, medir y registrar datos ambientales y mucho más), que los estudiantes controlan de forma remota desde cualquier navegador web (Department Of Education, Australian Government, 2020). La Facultad de Ingeniería de la UTS (Universidad Tecnológica de Sydney), en junio de 2001, decidió desarrollar cinco laboratorios de acceso remoto para estudiantes de pregrado de cursos de ingeniería (Lindsay et al., 2007). La UNSW (Universidad de Nueva Gales del Sur) desarrolló mecanismos para que los estudiantes de ingeniería eléctrica pudieran acceder a la misma experiencia práctica de laboratorio mientras aprenden de forma remota (UNSW, 2020).

Tres principales modelos se han utilizado como alternativa a la experiencia de sesiones presenciales de laboratorio: laboratorio casero, laboratorio en línea y kits de laboratorio enviados por paquetería.

El laboratorio casero consiste en diseñar experimentos en torno a lo que se puede encontrar fácilmente en casa. Esta metodología ahorra costos y hace que los experimentos sean más fáciles de identificar con el mundo que nos rodea. La desventaja es que algunos experimentos pueden requerir equipos o instrumentos especializados para tener un alcance o niveles de competencia específicos.

Con el laboratorio en línea, se intenta recrear la experiencia del laboratorio completamente en línea mediante la combinación de herramientas digitales como simuladores web y control remoto sobre equipos $\mathrm{y}$ condiciones de experimentación de un laboratorio a distancia, que se pueden operar desde la seguridad y comodidad del hogar de un estudiante. La mayor desventaja es que se elimina por completo el elemento "práctico". Se pierde la oportunidad de aprender a usar equipos y la adquisición de habilidades para ejecución de protocolos.

En el enfoque del modelo de experimento de kits de laboratorio por paquetería, éstos se envían directamente a los estudiantes para que realicen experimentos en su propia casa. Tiene la ventaja de brindar a los estudiantes una experiencia de laboratorio táctil sin límites de tiempo específicos establecidos sobre cuánto tiempo pueden aprender con el equipo. Se pueden presentar diversas desventajas como la pérdida o daño del kit durante su transporte o en la manipulación en casa, la logística y supervisión constante para el uso de los kits. Sin embargo, aún con estas desventajas, este enfoque de enseñanza es el que presenta mayores ventajas para los estudiantes y su aprendizaje práctico.

Comparando estas tres estrategias para la enseñanza de laboratorio durante la pandemia, se puede identificar que los kits para trabajar en casa representan la estrategia con mayor ventaja debido a la posibilidad de desarrollar habilidades de tipo experimental enfrentando al alumno con situaciones lo más cercano a la realidad en un laboratorio. Sin embargo, el costo de este tipo de kits puede llegar a ser muy elevado, por ejemplo, MX\$1,850.00, Scintia KIB: Kit de Introducción a Biotecnología (SCINTIA, 2021) y de MX\$3500.00, Odin kit: Genetically Engineer Any Brewing or Baking Yeast to Fluoresce (THE ODIN, 2021). Kits que contienen los elementos necesarios para la ejecución de protocolos específicos de ingeniería genética, pero el resto de las unidades de formación quedan fuera del diseño disponible por estas empresas. Por ello, en el Tecnológico de Monterrey (Campus Estado de México) se realizó un diseño de prácticas de laboratorio para adaptar a casa. Para lo cual fue necesario la elaboración de los instrumentos a emplearse

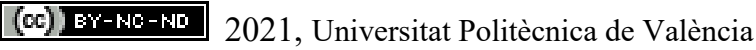

Congreso In-Red (2021) 
en los protocolos diseñados por medio de materiales de bajo costo, pero con vida media compatible con el uso a dársele al dispositivo. Coordinar la entrega personal de kits HomeLab con instrumentos, reactivos y materiales a estudiantes en condiciones de sana distancia y seguridad sanitaria.

Con la finalidad de desarrollar las competencias procedimentales de laboratorio en los estudiantes de Ingeniería en Biotecnología, del Tecnológico de Monterrey, Campus Estado de México, durante la pandemia COVID-19, los profesores a cargo diseñaron un kit de prácticas de biotecnología (HomeLab) para ejecución en casa. La propuesta HomeLab combina la supervisión a distancia en tiempo real, junto con el desarrollo de materiales multimedia, lo que permitió la conjugación de habilidades procedimentales con el manejo de los contenidos teóricos fundamentales en el desarrollo de las competencias de un Ingeniero en Biotecnología.

\section{Objetivos}

$\star$ Diseñar e implementar el uso kits para la elaboración de protocolos experimentales en el hogar ante contingencia COVID-19, estrategia denominada HomeLab.

$\star$ Construir y aplicar 5 dispositivos que permitieran ejecutar protocolos de un laboratorio de biotecnología y biocatálisis.

$\star$ Planear, ejecutar y evaluar el desarrollo de sesiones de ejecución experimental sincrónica con asesoramiento remoto.

$\star$ Diseñar e implementar diversas plataformas digitales como estrategias de evaluación y medios de vinculación profesor-estudiantes compatibles con el diseño HomeLab.

\section{Desarrollo de la Innovación}

\subsection{Perfil Participantes}

Los participantes son estudiantes que pertenecen a la carrera de Ingeniería en Biotecnología de $7 \mathrm{mo.}$ semestre. Los cuales fueron asignados en 4 grupos, con un número promedio de 16 estudiantes por grupo. Un 5\% de los estudiantes habían tomado previamente algún curso de laboratorio (Plan de estudios 2017). Sin embargo, el 95\% de los estudiantes con los que se aplicó la estrategia de aprendizaje HomeLab no tenía experiencia previa en laboratorios.

\subsection{Pertinencia de la Estrategia HomeLab en Programa Académico}

El programa académico Ingeniero en Biotecnología (Tecnológico de Monterrey, Plan 2017) consta de nueve semestres, siendo el séptimo, aquel en que los estudiantes cursan la concentración profesional denominada "Biocatálisis" la cual está diseñada para el desarrollo de 3 competencias disciplinares:

I. Desarrollar tecnologías y biosistemas utilizando información biológica o molecular con base en las necesidades de la industria y la sociedad.

II. Diseñar biorreactores capaces de sustentar las necesidades específicas de células en la generación de productos de interés.

III. Diseñar procesos de purificación de productos biotecnológicos con base en las especificaciones del mercado y en principios de sustentabilidad.

Las unidades de formación que conforman la concentración de biocatálisis contribuyen de manera integral al entendimiento de la función molecular de las proteínas y específicamente de enzimas, enriqueciendo el alcance funcional de estas a través de la ingeniería genética. Adicionalmente, se incorporan los 
conocimientos asociados a la manipulación y técnicas de propagación de diversos tipos de células como sistemas de expresión y los procesos asociados a su expresión, producción, y caracterización.

Como se puede observar en la tabla 1, existen aproximadamente 16 objetivos relacionados a competencias procedimentales que requerirían de un laboratorio para su adquisición a lo largo del semestre de la concentración de biocatálisis. Por tal motivo era imperante encontrar estrategias que permitieran a nuestros estudiantes adquirirlas aún con la carencia de un Laboratorio en donde desarrollaran prácticas experimentales y proyectos asociados. Por lo que el cuerpo docente se dio a la tarea del planteamiento de protocolos experimentales con posibilidad de adquisición de estas competencias desde el hogar de cada estudiante.

Tabla 1. Objetivos para cada unidad de formación de la concentración de biocatálisis.

\section{UNIDAD DE} FORMACIÓN
OBJETIVOS

"Al finalizar este curso el alumno será capaz de"

\begin{abstract}
Ingeniería Genética
Comprender el funcionamiento y analizar los procesos de manipulación genética que utilizan métodos de ADN recombinante, conocer los usos y aplicaciones de vectores para la clonación y la expresión de proteínas, explicar el diagnóstico genético para el análisis de organismos modificados genéticamente, enfermedades genéticas, disfunciones metabólicas e identificación de patógenos.
\end{abstract}

Laboratorio de Ingeniería Genética

Enzimología y

Biocatálisis

Laboratorio de enzimología y

biocatálisis

Cultivo de tejidos

Laboratorio de cultivo de tejidos

Laboratorio de

Bioprocesos

Ingeniería de proteínas
Desarrollar células competentes para capturar ADN, manipular vectores plasmídicos, analizar el ADN clonado y generar organismos modificados genéticamente, realizar identificaciones genéticas.
Aplicar conocimiento básico relacionado con el uso, análisis y recuperación de enzimas y los fundamentos para la estimación de modelos cinéticos.

Recuperar y purificar enzimas, determinar la actividad enzimática, aplicar el análisis cinético a datos experimentales y analizar el efecto de diferentes factores que afectan la acción enzimática.

Comprender el proceso de desarrollo de tejidos, analizar diversas técnicas para la propagación de tejidos, controlar el desarrollo y formación de órganos en un tejido, identificar la aplicación comercial de la micropropagación, diseñar ensayos biológicos para análisis de fármacos y fundamentar un modelo de negocio basado en cultivo de tejidos.

Aplicar diversos métodos para la clonación de plantas, implementar medios para la propagación de células vegetales y animales, promover el desarrollo de organismos vegetales completos a partir de células, obtener un cultivo primario para propagación de células animales en laboratorio y evaluar ensayos de citotoxicidad y viabilidad.

Utilizar los conocimientos en bioprocesos y tecnologías emergentes para llevar a cabo el diseño, operación y análisis de la operación de equipos utilizados en procesos de separación, concentración, purificación, y/o transformación de diversos biomateriales.

Comprender la relación entre la estructura molecular y la función de las proteínas, analizar el plegado y los motivos estructurales en las proteínas, entender la mutagénesis sitio dirigida y la mutagénesis al azar, aplicar bases de datos internacionales para el estudio de las proteínas y visualizar estructuras tridimensionales de proteínas.

\title{
3.3 Equipos para el desarrollo de las prácticas
}

Los equipos que se diseñaron para el desarrollo de las prácticas fueron los siguientes: mini lámpara de luz UV, mini transiluminador de luz azul, cámara de electroforesis, mini centrífuga (con velocidad no modulable de 13,000 rpm). Todos los equipos fueron construidos con materiales como acrílico, MDF y PLA para las piezas impresas (Fig. 1). 


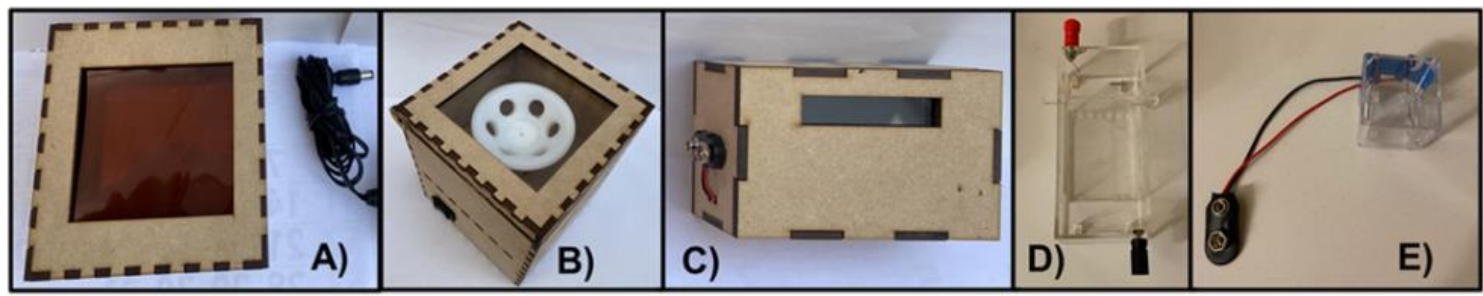

Fig. 1. Equipos diseñados y construidos para la realización de protocolos de biocatálisis. A) mini transiluminador de luz azul, B) Mini centrífuga, C) Minicámara de luz azul LED, D) Cámara de electroforesis, y E) cámara de electroelución.

\subsection{Reactivos para el desarrollo de las prácticas}

Los reactivos proporcionados para el desarrollo de las prácticas del laboratorio de genética fueron: $1 \mathrm{ml}$ Solución de Lisis (10 mM Tris-HCl pH 8.0, 0.5\% SDS, 5 mM EDTA), $500 \mu$ l Solución de acetato (Acetato de Amonio $8 \mathrm{M}$ y $1 \mathrm{mM}$ EDTA), $800 \mu \mathrm{l}$ de Isopropanol, $100 \mathrm{ml}$ de Solución fundida y gelificada de agarosa $0.8 \%$ con $0.3 \mathrm{X}$ SYBR gold, $300 \mathrm{ml}$ de buffer TBE $1 \mathrm{X}, 100 \mu \mathrm{l}$ de buffer de carga de ácidos nucleicos, 500 $\mu \mathrm{l}$ de agua grado biología molecular, $50 \mu \mathrm{l}$ de muestras de marcador de $\mathrm{pb}$ de $\mathrm{ADN}$, producto de PCR y plásmido; 1 placa de agar LB con ampicilina $(100 \mu \mathrm{g} / \mathrm{ml})$, células $E$. coli DH5 $\alpha$ liofilizadas, $1 \mathrm{ml}$ de medio de cultivo SOC, $100 \mu \mathrm{l}$ de plásmido J23100 (http://parts.igem.org/Part:BBa_J23100), 50 $\mu \mathrm{L}$ de buffer de lisis $(10 \mathrm{mM}$ de tris-HCl pH 8.0, $1 \mathrm{mM}$ EDTA, $0.1 \mathrm{mg} / \mathrm{mL}$ BSA, $0.2 \mathrm{mg} / \mathrm{mL}$ RNAsa A, sacarosa al $15 \%$ y $2 \mathrm{mg} / \mathrm{mL}$ de lisozima) y un fragmento de ADN embebido en agarosa $0.8 \%$ y teñido con SYBR-Green $1 \mathrm{X}$.

Los reactivos proporcionados para el desarrollo de las prácticas del laboratorio de enzimología fueron: 50 $\mathrm{mL}$ de acetona pura, $250 \mathrm{~mL}$ de agua bidestilada, $25 \mathrm{~mL}$ de solución de $\mathrm{NaOH} 0.1 \mathrm{M}, 25 \mathrm{~mL}$ de ácido acético $0.1 \mathrm{M}, 20 \mathrm{~mL}$ Buffer A ( $\mathrm{NaH}_{2} \mathrm{PO}_{4} 30 \mathrm{mM}, \mathrm{NaCl} 100 \mathrm{mM}$, Imidazol $20 \mathrm{mM}$ pH 8.0), $20 \mathrm{~mL}$ Buffer B ( $\mathrm{NaH}_{2} \mathrm{PO}_{4} 30 \mathrm{mM}, \mathrm{NaCl} 100 \mathrm{mM}$, Imidazol $300 \mathrm{mM} \mathrm{pH} \mathrm{8.0),} 250 \mathrm{~mL}$ de benzal, $20 \mathrm{~mL}$ de solución de Yodo, $25 \mathrm{~mL}$ de solución de $1.5 \mathrm{~mL}$ de solución de quimosina y pepsina (Cuajo), 2 cápsulas de amilasa comercial (suplemento alimenticio), $5 \mathrm{~mL}$ de solución de extracto proteico con GFP, $5 \mathrm{~mL}$ de extracto proteico con RFP, tubo cónico de $50 \mathrm{~mL}$ con $1 \mathrm{~g}$ de almidón, tubo cónico de $50 \mathrm{~mL}$ con $35 \mathrm{~g}$ de grenetina, $1 \mathrm{~mL}$ de resina cromatográfica de afinidad a $\mathrm{Ni}$.

Los reactivos proporcionados para el desarrollo de las prácticas del laboratorio de Bioprocesos incluyeron 2 tubos de vidrio de $10 \mathrm{~mL}$ con tapa de rosca, tiras reactivas de $\mathrm{pH}$, un tapón de silicón para frascos de 500 $\mathrm{mL}$, y un dispositivo airlock.

Los materiales utilizados para el desarrollo de las prácticas del laboratorio de Cultivo de Tejidos se indican a continuación. Se emplearon cajas de cultivo con medio Murashige Skoog, navajas estériles, colagenasa, medio de cultivo DMEM, buffer de fosfatos, placas de cultivo, fragmento de carne, semillas de lechuga, explantes de violeta africana y suculentas.

Todos los reactivos que no fueron enlistados fueron conseguidos por parte de los estudiantes y la mayoría de ellos son de uso común en el hogar (por ejemplo: detergente para trastes, alcohol, entre otros).

\subsection{Materiales para el desarrollo de las prácticas}

Se proporcionaron los siguientes materiales: Jeringas de 10, 5, 1 y $0.3 \mathrm{ml}$; gradilla para tubos cónicos, gradilla para microtubos, tubos cónicos de 15 y $50 \mathrm{ml}$, flotador, guantes, bolsas de plástico, Parafilm, cables para conexión banana-caimán, espátula Drigalski, escobillón, pipetas Pasteur, tira de $\mathrm{pH}$, mangueras plásticas flexibles,

Todos los materiales que no fueron enlistados fueron conseguidos por parte de los estudiantes, como por ejemplo un termómetro, lámpara de alcohol, recipientes, etc. 


\subsection{Diseño de Prácticas}

El orden y planeación de la estrategia de HomeLab, fue postulado y evaluado por los profesores de la concentración en conjunto. El diseño de prácticas para su implementación en casa implicó un análisis detallado de las competencias a desarrollar en cada unidad de formación, así como los conocimientos teóricos y prácticos asociados. En la tabla 2 se pueden observar las prácticas diseñadas, así como los medios de comunicación, simuladores complementarios, estrategias didácticas y recursos bioinformáticos empleados para la cobertura de los objetivos de aprendizaje de cada unidad de formación.

Tabla 2. Prácticas desarrolladas por laboratorio, mostrando los simuladores, herramientas y recursos didácticos empleados.

\begin{tabular}{|c|c|c|c|c|}
\hline Materia & Prácticas de HomeLab & Simuladores & $\begin{array}{l}\text { Herramientas } \\
\text { Bioinformática } \\
\text { s y bases de } \\
\text { datos }\end{array}$ & Recursos didácticos \\
\hline $\begin{array}{l}\text { Ingeniería } \\
\text { genética }\end{array}$ & $\begin{array}{l}\text { 1.-Extracción de ADN de } \\
\text { chícharos } \\
\text { 2.-Extracción de ADN de saliva } \\
\text { 3.-Electroforesis de ácidos } \\
\text { nucleicos } \\
\text { 4.-Transformación de bacterias } \\
\text { 5.-Clonación molecular } \\
\text { 6.-Diseño de primers } \\
\text { 7.-Extracción de ADN } \\
\text { plasmídico } \\
\text { 8.-Purificación de ácidos } \\
\text { nucleicos mediante } \\
\text { electroelución. }\end{array}$ & $\begin{array}{l}\text { Labster } \\
\text { 5.-Clonación } \\
\text { molecular }\end{array}$ & $\begin{array}{l}\text { NCBI } \\
\text { Uniprot } \\
\text { GeneCards } \\
\text { Benchling } \\
\text { Snapgene } \\
\text { Addgene } \\
\text { Labster } \\
\text { ExPASy }\end{array}$ & $\begin{array}{l}\text { Presentación Power Point } \\
\text { Video } \\
\text { Protocolo escrito del } \\
\text { desarrollo experimental } \\
\text { de las prácticas } \\
\text { Seguimiento remoto en } \\
\text { tiempo real } \\
\text { Flipgrid para discusión. } \\
\text { Google Sites para } \\
\text { elaboración de bitácoras }\end{array}$ \\
\hline $\begin{array}{l}\text { Enzimología e } \\
\text { Ingeniería de } \\
\text { proteínas }\end{array}$ & $\begin{array}{l}\text { 1.-Análisis de actividad catalasa } \\
\text { en extractos vegetales. } \\
\text { 2.-Extracción y Purificación de } \\
\text { enzimas a partir de residuos } \\
\text { agroindustriales. } \\
\text { 3.-Efecto de pH sobre actividad } \\
\text { de Amilasas. } \\
\text { 4.-Elaboración de Quesos y } \\
\text { determinación de fuerza del } \\
\text { cuajo } \\
\text { 5.-Purificación de proteína GFP } \\
\text { por Cromatografía de afinidad a } \\
\text { Níquel } \\
\text { 6.-Inmovilización de RFP por } \\
\text { atrapamiento. }\end{array}$ & $\begin{array}{l}\text { Labster } \\
\text { 1.-Cinética } \\
\text { Enzimática } \\
\text { 2.-Protein } \\
\text { Purification } \\
\text { Simulator } \\
\text { Faculty of Biological } \\
\text { Sciences University } \\
\text { of Leeds. } \\
\text { http://www.agbooth. } \\
\text { com/pp ajax/ } \\
\text { Purificación de } \\
\text { proteínas/SDS- } \\
\text { PAGE. }\end{array}$ & $\begin{array}{l}\text { PDB } \\
\text { UniProt } \\
\text { Swiss Modeler } \\
\text { I-tasser } \\
\text { RAMPAGE } \\
\text { Protein Sol } \\
\text { KEGG } \\
\text { BRENDA }\end{array}$ & $\begin{array}{l}\text { Presentación Power Point } \\
\text { con videos insertados de } \\
\text { ejecución demostrativa y } \\
\text { resultados a obtener. } \\
\text { Videos demostrativos de } \\
\text { la secuencia de ejecución } \\
\text { experimental. } \\
\text { Protocolo escrito del } \\
\text { desarrollo experimental } \\
\text { de las prácticas. } \\
\text { Flipgrid para discusión } \\
\text { de resultados. } \\
\text { Seguimiento remoto en } \\
\text { tiempo real. } \\
\text { Google Sites para } \\
\text { elaboración de bitácoras. }\end{array}$ \\
\hline Bioprocesos & $\begin{array}{l}\text { 1. Pasteurización de jugo de } \\
\text { naranja } \\
\text { 2. Fermentación alcohólica }\end{array}$ & $\begin{array}{l}\text { Labster } \\
\text { 1. Pasteurización } \\
\text { 2. Crecimiento } \\
\text { microbiano } \\
\text { 3. Recristalización: } \\
\text { Purifica tu } \\
\text { compuesto. }\end{array}$ & PubMed & $\begin{array}{l}\text { Presentación Power Point } \\
\text { Protocolo escrito } \\
\text { Videos demostrativos de } \\
\text { la secuencia de ejecución } \\
\text { experimental } \\
\text { Flipgrid para discusión } \\
\text { de resultados. } \\
\text { Seguimiento remoto en } \\
\text { tiempo real. } \\
\text { Google Sites para } \\
\text { elaboración de bitácoras } \\
\text { Reporte escrito }\end{array}$ \\
\hline $\begin{array}{l}\text { Cultivo de } \\
\text { tejidos }\end{array}$ & 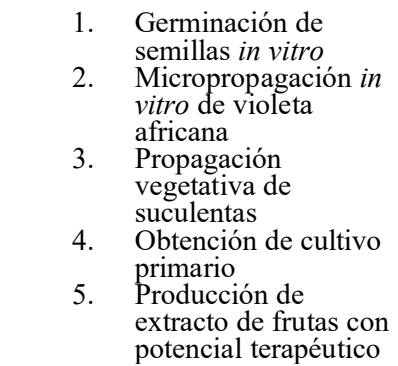 & Labster & PubMed & $\begin{array}{l}\text { Presentación Power Point } \\
\text { Protocolo escrito } \\
\text { Video demostrativo de la } \\
\text { ejecución demostrativa } \\
\text { de la secuencia } \\
\text { experimental } \\
\text { Discusión de resultados } \\
\text { obtenidos mediante } \\
\text { Flipgrid } \\
\text { Difusión científica de } \\
\text { fundamentos y resultados } \\
\text { a través de Tik Tok. } \\
\text { Reporte escrito }\end{array}$ \\
\hline
\end{tabular}


Ana Laura'Torres-Huerta, Rigel V. Gómez-Acata, Manuel Jaime-Rodríguez, Yolanda. G. García-Huante, Jesús I. Calzada-Frías, Elías O. Gómez-Montes, Roberto Delgado-Durán, César García-Díaz, Carlos E. Gómez-Sánchezi, Aurora Antonio-Pérez.

Todas las prácticas contaron con un protocolo previamente probado por los docentes. Sin embargo, esto no limita el aprendizaje o el análisis que pueden hacer los estudiantes de sus propios resultados. Es decir, en algunas ocasiones, los resultados obtenidos no coinciden con los esperados. En estos casos, se solicitó al estudiante un análisis de las condiciones ambientales en las que realizó su práctica y en particular cada uno de los experimentos para explicar las posibles circunstancias de la variación de sus resultados.

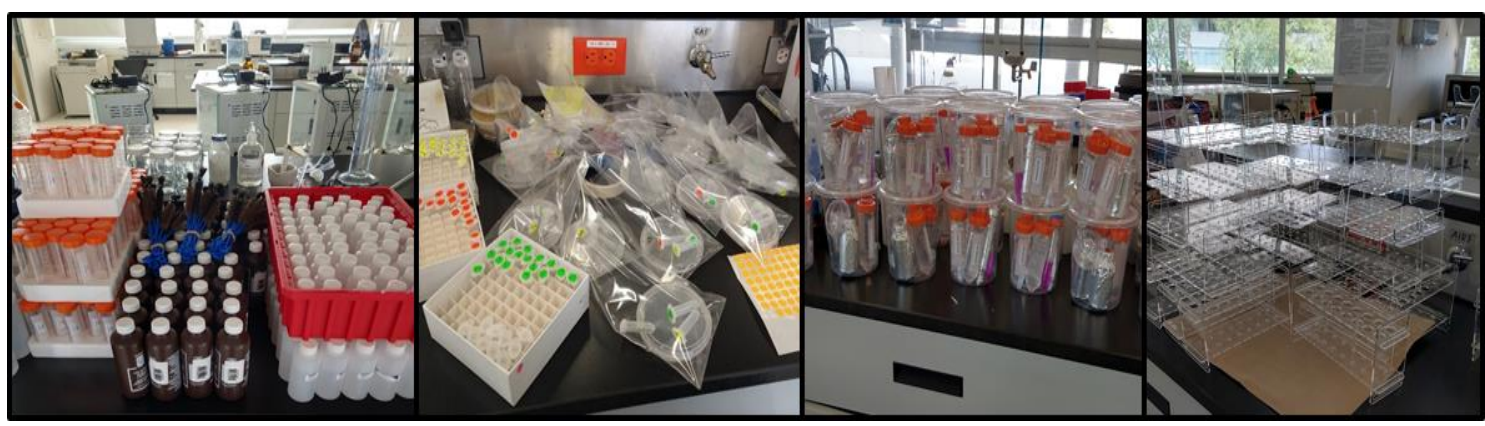

Fig. 2. Materiales y reactivos preparados para la ejecución de protocolos de biocatálisis. Incluyendo como muestras representativas, sustratos y enzimas, amortiguadores, placas de cultivo, células liofilizadas, proteinas purificadas, resinas cromatográficas, gradillas.

\subsection{Plataformas de interacción}

Las plataformas de interacción estudiante-profesor utilizadas se especifican en la Tabla 3.

Tabla 3. Medios y plataforma de interacción profesor-estudiante

\begin{tabular}{|c|c|c|}
\hline Plataforma & Descripción & Actividad ejecutada \\
\hline Zoom & $\begin{array}{l}\text { Aplicación de videollamadas disponible en } \\
\text { Windows, Mac, iOS, Android y Linux. }\end{array}$ & $\begin{array}{l}\text { Canal de comunicación sincrónica principal para el } \\
\text { desarrollo de todas las sesiones, medio de grabación pa } \\
\text { consulta asincrónica del curso. }\end{array}$ \\
\hline CANVAS & $\begin{array}{l}\text { Plataforma Open Source de e-learning (LMS) } \\
\text { para gestionar clases online. }\end{array}$ & $\begin{array}{l}\text { Gestión de clases online, reservorio de material didácti } \\
\text { medio de comunicación, programador y espacio reservo } \\
\text { de entrega de evidencias de aprendizaje. }\end{array}$ \\
\hline FlipGrid & $\begin{array}{l}\text { Aplicación donde los estudiantes graban vídeos } \\
\text { de duración variable, desde } 15 \text { segundos, a } 10 \\
\text { minutos. }\end{array}$ & $\begin{array}{l}\text { Permitió valorar y desarrollar competencias comunicat } \\
\text { conceptuales y hasta actitudinales de forma individual. } \\
\text { estudiantes desarrollaron pequeños videos vinculados a } \\
\text { sesiones de discusión ejecutadas en grupo previamente }\end{array}$ \\
\hline Google Sites & $\begin{array}{l}\text { Aplicación online que permite crear un sitio } \\
\text { web de grupo de una forma sencilla. }\end{array}$ & $\begin{array}{l}\text { Los estudiantes reunieron un único espacio y de una fo } \\
\text { rápida información relacionada a su ejecución experim } \\
\text { a manera de Bitácora de laboratorio donde pudieron in } \\
\text { vídeos de su ejecución, registros fotográficos, } \\
\text { presentaciones, archivos adjuntos y texto. }\end{array}$ \\
\hline YouTube & Sitio web dedicado a compartir videos. & Reservorio y fuente de material videográfico \\
\hline TikTok & $\begin{array}{l}\text { Aplicación para compartir videos que permite } \\
\text { crear y compartir videos de } 15 \text { segundos. }\end{array}$ & $\begin{array}{l}\text { Generación de videos por parte de los estudiantes para } \\
\text { evidenciar la comprensión de protocolos y resultados. }\end{array}$ \\
\hline
\end{tabular}




\subsection{Desarrollo de sesiones}

Todas las prácticas fueron implementadas por los estudiantes en sus casas bajo la supervisión sincrónica en línea de los profesores junto con apoyo de diversas herramientas como videos, simuladores y aplicación de mensajería instantánea. Las sesiones experimentales fueron programadas con un tiempo de 3 horas para cada uno de los laboratorios por semana, divididas en dos sesiones de 90 minutos.

El esquema general de trabajo para cada práctica consistió en 2-4 sesiones en las que se realizó:

1. Calendarización de actividades incluidas en el desarrollo de las prácticas (Indicaciones técnicas y de fechas para sesión introductoria, experimental y discusión de resultados).

2. Entrega previa de protocolos experimentales.

3. Revisión de protocolos experimentales por parte de los estudiantes.

4. Evaluación en línea de la lectura previa de los protocolos experimentales.

5. Sesión introductoria de contenidos asociados a la práctica.

6. Indicaciones técnicas específicas para la ejecución experimental (uso de material videográfico), manejo de equipos, toma de muestras y disposición de desechos.

7. Sesiones experimentales supervisadas en tiempo real, con la generación de bitácoras por parte de los alumnos, para la documentación de evidencias de trabajo experimental.

8. Sesión de discusión de resultados y cierre de la práctica.

\subsection{Herramientas de Evaluación}

La evaluación de cada periodo experimental que comprende el desarrollo y comprensión de 3-4 prácticas guiadas incluyó:

- Ejecución experimental de los protocolos, lo que conduce al desarrollo de habilidades técnicas (research based, research tutored), supervisada vía Zoom de forma sincrónica.

- Análisis de resultados, que conlleva el contraste de los resultados obtenidos con los de la literatura, por lo que los estudiantes hacen una exhaustiva búsqueda bibliográfica y hacen el análisis de los resultados de otros autores (research oriented). Ejecutada en forma asincrónica y posteriormente de forma colaborativa estudiantes-docente en Zoom de forma sincrónica.

- Participación en la sesión de discusión de resultados en forma verbal durante la defensa de sus resultados experimentales (research tutored), ejecutada en Zoom de forma sincrónica.

- Reporte escrito que incluía: resumen, justificación, resultados, discusión, conclusión y bibliografía. Entregado por estudiantes y retroalimentado en plataforma CANVAS.

- Bitácora de laboratorio elaborada en Google Sites de forma colaborativa y constante a lo largo de las diversas sesiones. Conteniendo evidencias videográficas, fotográficas y documentales.

- Argumentación individual elaborada y registrada vía FlipGrid.

\subsection{Encuesta De Evaluación Estrategia HomeLab}

La encuesta de evaluación de la estrategia HomeLab consistió en 19 preguntas cerradas, aplicada a un total de 40 encuestados, que corresponde al $76 \%$ de los estudiantes que realizaron sus prácticas con el kit HomeLab en el periodo agosto a diciembre del año 2020.

Los cuestionamientos planteados fueron relacionados a:

- Logística de la estrategia de entrega.

- Desempeño de la facultad. 
Ana Laura 'Torres-Huerta, Rigel V. Gómez-Acata, Manuel Jaime-Rodríguez, Yolanda. G. García-Huante, Jesús I. Calzada-Frías, Elías O. Gómez-Montes, Roberto Delgado-Durán, César García-Díaz, Carlos E. Gómez-Sánchezi, Aurora Antonio-Pérez.

- Satisfacción de los estudiantes.

- Eficacia de los recursos didácticos en la estrategia HomeLab.

- Evaluación de la adquisición de competencias.

- Valoración emocional de los estudiantes durante el desarrollo de la estrategia HomeLab.

\section{Resultados}

\subsection{Diseño de Prácticas}

Como parte del diseño de los kits, los ensayos experimentales de cada práctica de laboratorio se verificaron antes de su entrega a los estudiantes, con la finalidad de validar la funcionalidad de sus materiales, equipos y la precisión de los protocolos. Además, los profesores se encargaron de desarrollar el material multimedia de apoyo (videotutoriales), así como probar el uso de múltiples aplicaciones. Sin embargo, durante la ejecución de las prácticas por parte de los estudiantes, los resultados presentaron variaciones, desde los cercanos a lo esperado y algunos negativos. Entre los factores que más influyeron en estas variaciones se encuentra la dificultad para utilizar materiales y equipos por primera vez, adecuación de un espacio de trabajo de laboratorio en casa, seguimiento a las instrucciones del profesor y falta de experiencia en la ejecución de los protocolos experimentales.

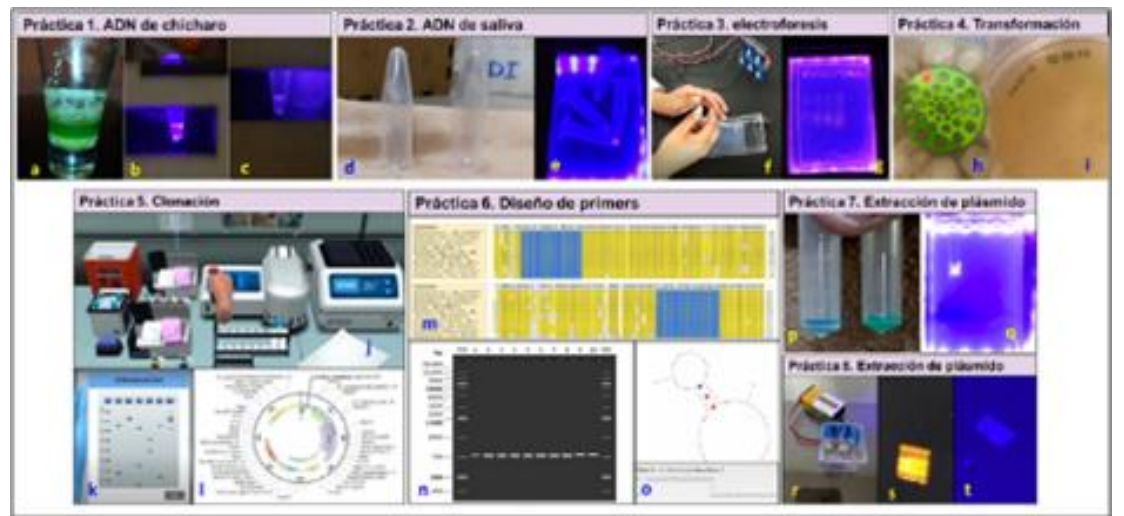

Fig. 3. Evidencia de resultados de las prácticas de laboratorio de ingeniería genética. Se muestran figuras representativas de resultados obtenidos por los estudiantes en las ocho prácticas.
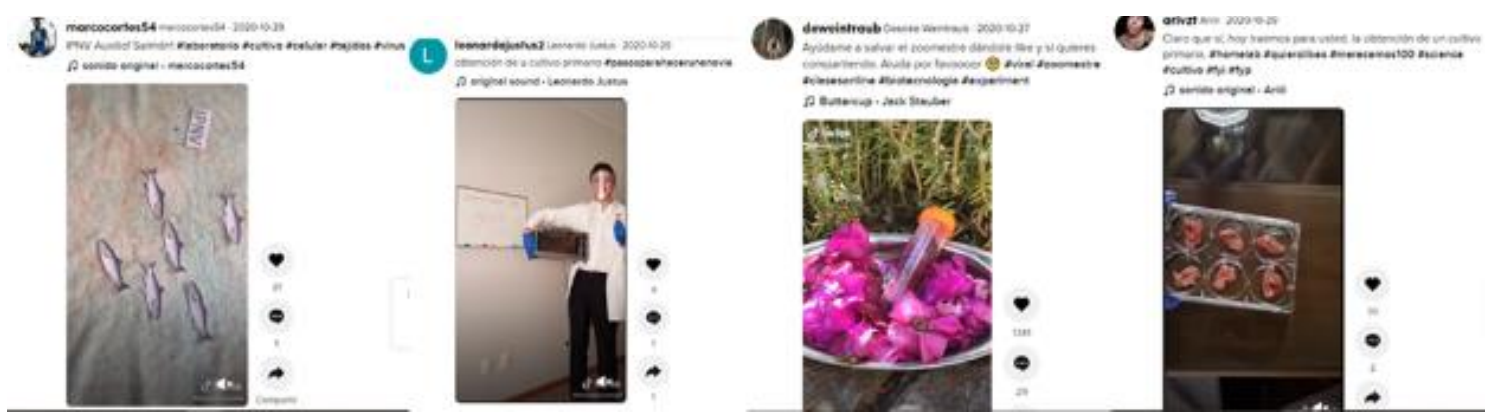

Fig. 4. Evidencia de Tik Tok realizados por los alumnos durante las prácticas de cultivo de tejidos. Se comprobó que mediante plataformas con las cuales los estudiantes están familiarizados, se puede evidenciar la comprensión de las prácticas de forma eficaz.

En las figuras 3-6 se pueden observar ejemplos reales de estudiantes de resultados esperados para las prácticas de los distintos laboratorios. La mayor parte de las prácticas presentaron éxito en cuanto a la obtención del resultado esperado, salvo algunas excepciones, como en el caso del Laboratorio de Ingeniería 
Genética donde las muestras de ácidos nucleicos presentaron degradación por las condiciones de envío y almacenamiento.

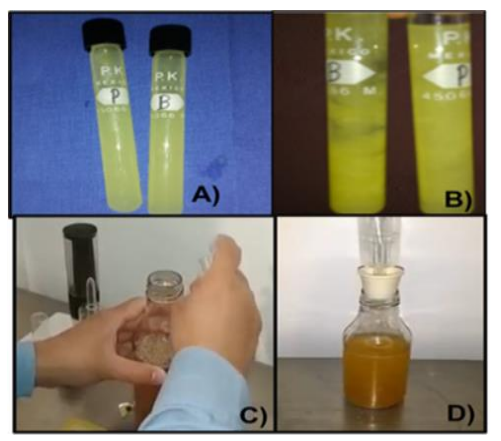

Fig.5. Evidencia de los resultados obtenidos en las prácticas de bioprocesos. A) y B) pasteurización del jugo de naranja, antes y después del proceso térmico, C) proceso de inoculación del extracto de malta con la levadura liofilizada, D) equipo de fermentación con airlock.

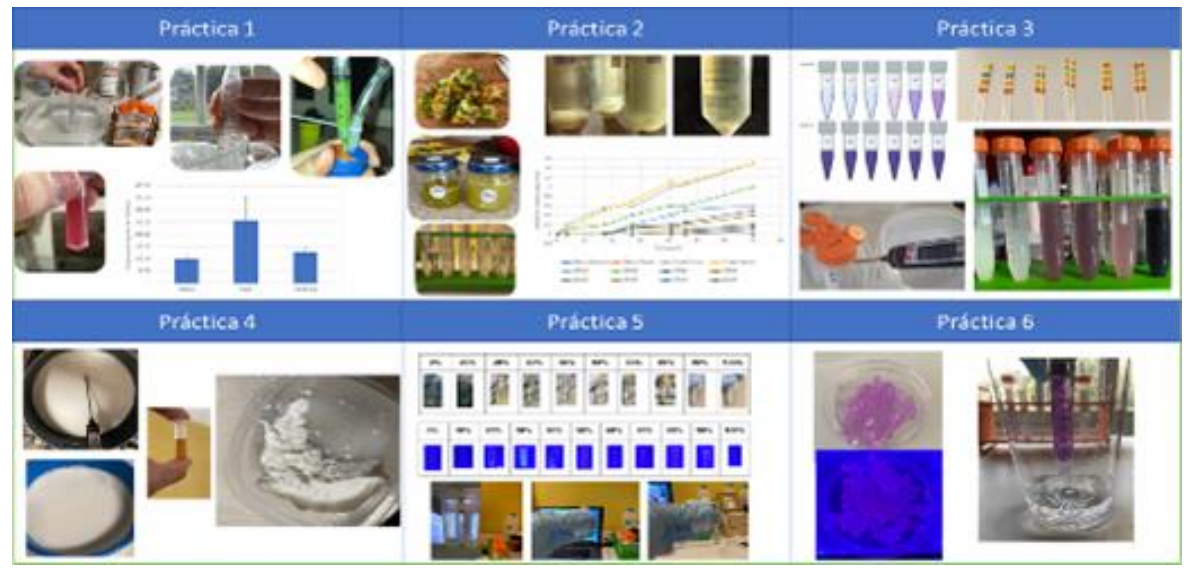

Fig.6.- Evidencia de resultados de las prácticas de laboratorio de ingeniería genética. Se muestran figuras representativas de resultados obtenidos por los estudiantes en las ocho prácticas.

\subsection{Resultados Encuesta}

Respecto a las estrategias didácticas que se emplearon para transmitir instrucciones de ejecución, conceptos teóricos y técnicos asociados al desarrollo de las prácticas, el $72 \%$ los estudiantes indicaron que las instrucciones proporcionadas a través de protocolos escritos, videos demostrativos de ejecuciones experimental en sesiones introductorias al trabajo experimental le proporcionaron información suficiente de aspectos generalizados y específicos para los experimentales programados. Sólo el 18\% indicó que consideró que la información fue reducida en algunos aspectos y un 10\% consideró que la información proporcionada fue insuficiente de forma general, dificultando su ejecución en casa.

De los diversos formatos de comunicación e interacción empleados, los videos pregrabados, las presentaciones con videos incrustados e instrucción sincrónica en tiempo real fueron aquellos reconocidos como herramientas atractivas, específicas y dinámicas. que les permitieron una adquisición eficaz y precisa de conocimientos y capacidades procedimentales. 
Con respecto al estado anímico de los estudiantes durante las sesiones de ejecución experimental sincrónica con asesoramiento remoto, expresaron haber experimentado de forma preponderante confianza, interés, curiosidad y concentración. En menor proporción expresaron sensaciones como desconcierto, desesperación e inseguridad. No obstante, acorde a la estrategia didáctica, era necesario que los estudiantes revisaran previamente a la sesión experimental protocolos y videos demostrativos. El 50\%, 40\% y 10\% indicaron ejecutar estas actividades de forma constante, regular y ocasional respectivamente. Estas métricas podrían explicar la variabilidad de su estado anímico.

Por otro lado, el desarrollo de la sesión experimental sincrónica con asesoramiento remoto en tiempo real, el 78\% consideró que las instrucciones dadas durante la ejecución de los experimentales fueron abundantes y específicas, así como correspondieron a las inquietudes expresadas por la mayoría del grupo. Asociado a las responsabilidades de los estudiantes durante la sesión sincrónica alrededor del 95\% indicó que siempre o casi siempre estuvieron atentos a las instrucciones, consejos y comentarios emitidos por parte de los profesores en el asesoramiento remoto de los experimentos ejecutados.

A pesar de sensaciones vinculadas a aspectos negativos, alrededor del $80 \%$ de los estudiantes indicaron que ante dificultades o anomalías experimentales se sintieron con la capacidad y tranquilidad de resolverlas en medida que se desarrollaban los experimentales por su cuenta y con apoyo del profesor a cargo. El $96 \%$ indicó que pudieron analizar las variables, que afectaron sus resultados, así como mecanismos que involucran estas variables. Este cuestionamiento surgió a partir de que inevitablemente en sesiones de laboratorio presencial suceden eventualidades en la ejecución de las prácticas, los docentes esperaban fuesen más frecuentes con un asesoramiento remoto. De manera asociada esta capacidad de resolver los problemas que enfrentarían de forma remota, se les solicitó hacer una autoevaluación al respecto de su autonomía en la ejecución de las prácticas. Un $80 \%$ de los estudiantes identificaron su evolución constante a lo largo del curso, declarando que poco a poco comenzaron a experimentar seguridad en la toma de decisiones en ejecución experimental.

El 90\% indicó que al finalizar las sesiones de discusión de las prácticas de laboratorio pudo reflexionar y adquirir los conocimientos asociados al desarrollo de las prácticas y casi el 100\% consideró que era complejo obtener resultados idénticos por las variables que estaban correlacionadas a las condiciones de cada ejecución de cada integrante del grupo.

Finalmente, el 50\% estudiantes posicionaron a la estrategia de HomeLab como aquello que cumplió en mayor medida sus necesidades/expectativas de aprendizaje de laboratorio para la adquisición de competencias, habilidades y conocimientos en mientras que sólo un $8 \%$ señaló mayor satisfacción con el uso de simuladores y un $42 \%$ con la combinación de ambos métodos. No obstante, de manera particular el $60 \%$ de los estudiantes indicó que la estrategia HomeLab desarrolló más su capacidad de análisis deductivo contra un $10 \%$ que lo atribuyó al uso de simuladores.

\subsection{Competencias adquiridas}

En la Tabla 4 se pueden observar las prácticas ejecutadas, así como los objetivos de aprendizaje cubiertos asociados a cada una de ellas.

A través de los diversos medios de interacción, sesiones sincrónicas con supervisión en prácticas guiadas, sesiones de discusión y entregables planteados, se observó que: 
Tabla 4. Prácticas diseñadas y objetivos de aprendizaje cubiertos por los estudiantes en cada unidad de formación de la concentración de biocatálisis de la carrera Ingeniería en Biotecnología.

$\begin{array}{lll}\text { Unidad de } & \text { Práctica } & \text { Objetivos de Aprendizaje Cubiertos } \\ \text { Formación } & \end{array}$

Ingeniería $\quad 1 . \quad$ Extracción de ADN de chícharos.

Genética 2. Extracción de ADN de saliva.

3. Electroforesis de ácidos nucleicos.

4. Transformación de bacterias.

5. Clonación molecular.

6. Diseño de primers.

7. Extracción de ADN plasmídico.

8. Purificación de ácidos nucleicos mediante electroelución.

Enzimología

1. Análisis de actividad de catalasas vegetales.

2. Extracción y purificación de enzimas a partir de residuos agroindustriales.

3. Efecto de $\mathrm{pH}$ sobre actividad de Amilasas.

4. Elaboración de quesos

5. Determinación de parámetros cinéticos.

6. Purificación de proteína GFP por IMAC.

7. Inmovilización de RFP por atrapamiento.

Bioprocesos 1. Esterilización y Pasteurización.

2. Crecimiento Microbiano.

3. Fermentación alcohólica.

4. Lisis celular.

5. Determinación de kLa.

6. Extracción Líquido-Líquido.

7. Procesos de membrana: Ultrafiltración.

8. Cristalización.
1. Aislamiento de ácidos nucleicos y su caracterización (Prácticas $1,2$ y 7$)$

2. Evaluar integridad de ácidos nucleicos (Práctica 3).

3. Transformar bacterias y verificar la expresión de proteínas fluorescentes (Práctica 4)

4. Diseñar primers para amplificar genes de interés y realizar construcciones genéticas en plásmidos (Prácticas 5 y 6 ).

5. Purificar ADN mediante electroelución (Práctica 8).

1. Recuperar y purificar enzimas (Prácticas 2, 6 y 7)

2. Determinar la actividad enzimática (Prácticas 1, 2,3 y 4).

3. Aplicar el análisis cinético a datos experimentales (Práctica 5).

4. Analizar el efecto de diferentes factores que afectan la acción enzimática (Prácticas 3 y 4).

$\begin{array}{lll}\text { Cultivo de } & \text { 1. } & \text { Técnica aséptica. } \\ \text { tejidos } & \text { 2. } & \text { Monitoreo ambiental. } \\ \text { 3. } & \text { Preparación de medios de cultivo. } \\ \text { 4. } & \text { Mantenimiento de líneas celulares. } \\ \text { 5. } & \text { Conteo celular. } \\ \text { 6. } & \text { Modificación genética nuclear de plantas. } \\ \text { 7. } & \text { Cultivo en suspensión de raíces de } \\ & & \text { tomate. }\end{array}$

1. Conocer y aprender los principios básicos de las operaciones unitarias comúnmente utilizadas en bioprocesos (Prácticas 1, 3, $4,6,7,8)$.

2. Conocer y aprender diferentes métodos para la determinación del crecimiento microbiano (Práctica 2 y 3 ).

3. Aplicar el conocimiento adquirido en clase para ejecutar una fermentación para la producción de cerveza (Práctica 3).

4. Conocer los diferentes métodos para llevar a cabo la ruptura celular y/o de una matriz biológica (Práctica 4).

5. Conocer los métodos experimentales y ejecutar los cálculos para determinar el kLa de un biorreactor (Práctica 5).

6. Conocer diversas técnicas de recuperación primaria empleadas en el diseño de procesos biotecnológicos (Práctica 6 y 7).

7. Conocer las técnicas de purificación utilizadas comúnmente en el diseño de procesos biotecnológicos y las condiciones de operación (Práctica 6, 7 y 8).

1. Conocer las buenas prácticas para evitar la contaminación microbiológica de cultivos (Práctica 1, 3, 4, 7).

2. Medir el contenido microbiológico del aire y superficies de áreas controladas (Práctica 2).

3. Generar el ambiente idóneo de crecimiento celular a través de la combinación de distintos nutrientes y aditivos (Práctica 3).

4. Mantener las condiciones óptimas de crecimiento de células de mamífero en un laboratorio (Práctica 4).

5. Determinar la densidad y viabilidad celular de un cultivo para evaluar su estado y hacer experimentos reproducibles (Práctica $5)$.

6. Conocer las técnicas de expresión de GFP en plantas (Práctica 6).

7. Implementar el cultivo de raíces de tomate (Práctica 7). 
Ana Laura 'Torres-Huerta, Rigel V. Gómez-Acata, Manuel Jaime-Rodríguez, Yolanda. G. García-Huante, Jesús I. Calzada-Frías, Elías O. Gómez-Montes, Roberto Delgado-Durán, César García-Díaz, Carlos E. Gómez-Sánchezi, Aurora Antonio-Pérez.

- Los alumnos autogestionaron el desarrollo de sus protocolos experimentales, así como demostraron un progreso continuo en su autonomía en la toma de decisiones.

- Los alumnos desarrollaron competencias procedimentales particulares a cada laboratorio, durante la ejecución de los protocolos establecidos para su trabajo experimental en casa.

- Los alumnos documentaron sus ensayos experimentales en casa, acorde a los lineamientos estandarizados para la elaboración de bitácoras de laboratorio.

- Los alumnos compararon sus resultados con los obtenidos por sus compañeros y con lo reportado en la literatura, validando o refutando sus propios resultados y analizando sus procedimientos experimentales.

En la figura 7 se muestra un collage de algunos estudiantes ejecutando las prácticas con asistencia remota en tiempo real.

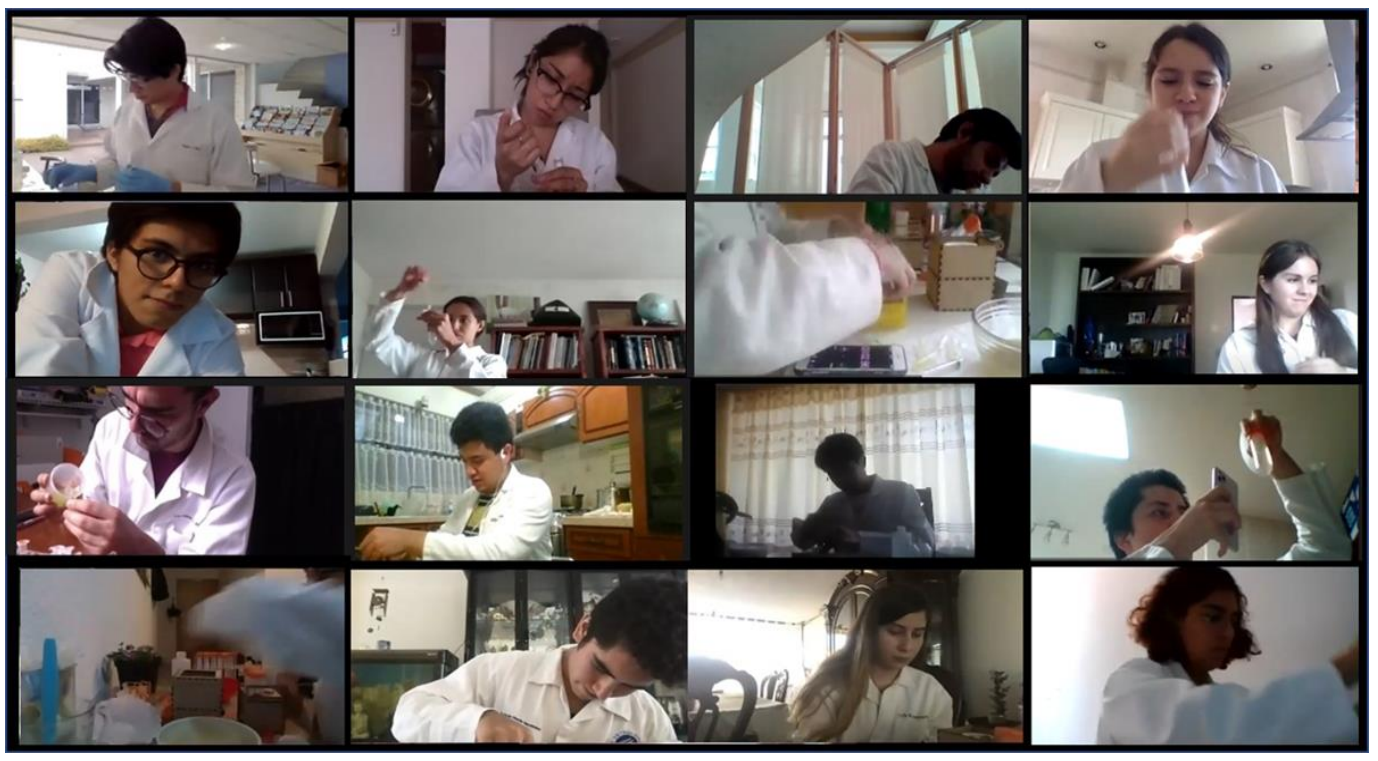

Fig.7.- Evidencia de ejecución de las prácticas de laboratorio. Se muestran fotografias representativas de algunos estudiantes en plataforma Zoom durante la ejecución de prácticas con asesoramiento remoto en tiempo real.

\section{Conclusiones}

Aunque este proyecto centra su análisis en el desarrollo de los elementos que constituyeron la propuesta de la estrategia HomeLab diseñada por los profesores involucrados en la concentración de biocatálisis desarrollada en el Tecnológico de Monterrey, campus Estado de México, como respuesta ante la situación actual de la pandemia por COVID-19, consideramos que sus resultados pueden ser de interés para otras universidades.

Aunque la enseñanza en línea tiene sus fortalezas, no podría proporcionar a los estudiantes la experiencia práctica en la realización de experimentos biológicos reales para dominar las habilidades técnicas; que son esenciales para que los estudiantes al egresar se desempeñen adecuadamente en un entorno laboral. Particularmente, 92\% estudiantes posicionaron a la estrategia de HomeLab como aquella que cumplió en mayor medida sus necesidades y expectativas de adquisición de habilidades técnicas y desarrollo de pensamiento deductivo, mientras que sólo un $8 \%$ correlacionó la adquisición de estas competencias al uso exclusivo de simuladores. 
Con el desarrollo de experimentos ejecutados en casa, los estudiantes pudieron ejecutar técnicas básicas de la biología molecular, protocolos de purificación de proteínas, montaje de ensayos enzimáticos, practicar principios de operaciones unitarias y bioprocesos; así como el desarrollo de cultivo de células vegetales. Adicionalmente, se fomentó el manejo de equipos especializados en una versión simplificada que se adaptó para usar en casa, más del $90 \%$ de alumnos se sintió con los conocimientos básicos y la confianza y familiarización suficiente para la manipulación de equipos en un entorno laboral a futuro.

Por otra parte, al enfrentarse nuestros alumnos a la ejecución real de sus experimentales con la guía del profesor de forma remota, $80 \%$ de ellos identificaron la evolución de su propia autogestión y autonomía en el desarrollo de su experimentales, permitiéndoles adquirir habilidades asociadas a la administración de tiempo, toma de decisiones, organización y tolerancia a la frustración.

Por este motivo pensamos que tanto la estrategia descrita y analizada en este trabajo, así como los puntos fuertes o débiles señalados en esta indagación, pueden ser de interés para profesionales y gestores implicados en procesos docentes universitarios ante esta nueva normalidad. Por lo que los profesores participantes estamos en disponibilidad de ofrecer asesoría o colaboración en la implementación de esta estrategia.

\section{Referencias}

BURKI, T. K. (2020). “COVID-19: consequences for higher education”. In The Lancet. Oncology (Vol. 21, Issue 6, p. 758). NLM (Medline). doi: 10.1016/S1470-2045(20)30287-4

DEPARTMENT OF EDUCATION, AUSTRALIAN GOVERNMENT. Freely Accessible Remote Laboratories (FARLabs). (2020). Retrieved from <https://www.farlabs.edu.au/info/about/> [consulta: 20 de marzo de 2021]

GLIDDON, C. M., \& J. ROSENGREN, R. (2012). “A laboratory course for teaching laboratory techniques, experimental design, statistical analysis, and peer review process to undergraduate science students". Biochemistry and Molecular Biology Education, 40(6), 364-371. doi: 10.1002/bmb.20645

LINDSAY, E., LIU, D., MURRAY, S., \& LOWE, D. (2007). "Remote laboratories in Engineering Education: Trends in Students' Perceptions", 18th Conference of the Australasian Association for Engineering Education, 09 Dec 2007; Melbourne, Australia: Australasian Association for Engineering Education.

MONASH UNIVERSITY. Teaching and learning in the COVID-19 era - Faculty of Pharmacy and Pharmaceutical Sciences, (2020). Retrieved from $<$ https://www.monash.edu/pharm/future/courses/pharmacy/teaching-and-learningin-the-covid-19-era> [consulta 20 de marzo de 2021].

RAY, S., \& SRIVASTAVA, S. (2020). "Virtualization of science education: a lesson from the COVID-19 pandemic". Journal of Proteins and Proteomics, 11(2), 77-80. doi: 10.1007/s42485-020-00038-7

SCINTIA. Biology Classes Reinvented, (2021). Retrieved from $<$ https://en.scintia.com/shop $>$ [consulta 21 de marzo de 2021].

THE ODIN. Genetically Engineer Any Brewing or Baking Yeast to Fluoresce, (2021). Retrieved from $<$ https://www.the-odin.com/ge-yeast/> [consulta 22 de marzo de 2021]

UNSW (UNIVERSITY OF NEW SOUTH WALES). Reimagining education from the lab to the living room Inside $U N S W$, (2020). Retrieved from $<$ https://www.inside.unsw.edu.au/innovation-and-engagement/reimaginingeducation-the-lab-the-living-room $>$ [consulta 20 de marzo de 2021]. 